\title{
LA REFLEXIÓN Y LA AUTO-EVALUACIÓN EN LA TRANSFORMACIÓN DE LOS PROCESOS DE EDUCACIÓN AMBIENTAL: ESTUDIO DE UN CASO EN EL JARDÍN BOTÁNICO DE BOGOTÁ
}

\author{
JULIO CÉSAR TOVAR-GÁLVEZ \\ joule_tg@yahoo.com
}

Manizales, 2010-11-26 (Rev. 2011-03-20)

RESUMEN

El reporte que se hace a través de este artículo se constituye como un caso analizado a través de una perspectiva cualitativa, mediante la cual se recupera información que da cuenta de las vivencias y formas de entender la educación ambiental de un grupo de profesionales que tiene diversas formas de aproximación a las comunidades de Bogotá. Las reflexiones y propuestas presentadas nacen a través de un proceso de formación y de acompañamiento a los participantes del Seminario Permanente de Formación de Formadores Ambientales Enrique Pérez Arbeláez. La información cualitativa, emergente de los espacios de discusión entre los participantes y el orientador, recogida por medio de estrategias como el diario de campo y guías de registro, evidencia la reflexión y la auto-evaluación como alternativas para estructurar la educación ambiental desde las particularidades de las comunidades de Bogotá

\section{PALABRAS CLAVE:}

Educación ambiental, reflexión, auto-evaluación, transformación, Jardín Botánico de Bogotá

REFLECTION AND SELF-EVALUATION IN THE TRANSFORMATION OF THE ENVIRONMENTAL EDUCATION PROCESS: A CASE STUDY IN THE BOGOTÁ BOTANICAL GARDEN

\section{ABSTRACT}

The report presented through this article becomes a case analyzed through a qualitative approach through which information accounting for the experiences and ways to understand environmental education of a group of professionals having a diverse approach to the communities in Bogotá. The reflections and proposals presented come from an educational and accompaniment process of the participants in the Environmental Formative Influence Permanent Education Seminar "Enrique Pérez Arbeláez". The qualitative information emerging from de discussion spaces between the participants and the presenter gathered using strategies such as field journals and registry guides evidences the reflection and self-evaluation as alternatives to structure environmental education from the particularities of the communities in Bogotá.

KEY WORDS: environmental education, reflection, self-evaluation, transformation, Bogotá Botanical Garden.

\section{INTRODUCCIÓN}

El presente artículo tiene como objetivo presentar parte del proceso de transformación de las propuestas de educación ambiental, que se está dando en uno de los equipos del Jardín Botánico de Bogotá -JBB- que realizan trabajo con las comunidades de la ciudad. En este sentido se rescata, en gran medida, la información que da cuenta de cómo un equipo de profesionales de la Subdirección Científica, apoyado por un profesional de la Subdirección Educativa y autor del presente texto, inician la reflexión en torno a lo que puede ser la educación ambiental para la ciudadanía, dirigiéndose hacia la auto-evaluación y transformación de los espacios de educación ambiental que ofrecen, en el marco de la misión de conservación y uso sostenible de la flora que la institución tiene frente a Bogotá.

Para comprender parte del contexto que da sentido a la información y análisis que se reportan más adelante sobre el caso a estudiar, es necesario enunciar que el Jardín Botánico de Bogotá José Celestino Mutis es una entidad pública que tiene como misión contribuir "[...] a la conservación de la flora del Distrito Capital, a la sostenibilidad ambiental de su territorio y al aprovechamiento de su patrimonio genético, a través de la investigación científica, la transferencia tecnológica y la educación ambiental" (JBB, n.f.). A partir de tal horizonte, la institución se organiza básicamente en 
tres dependencias: Subdirección Técnica Operativa (con proyectos como Arborización Urbana y Agricultura Urbana), Subdirección Científica (con proyectos como Restauración Ecológica, Conservación in situ y Uso Sostenible) y Subdirección Educativa y Cultural (con proyectos como Formación de Líderes Ambientales y Formación de Gestores Ambientales); las cuales tienen múltiples procesos y varias líneas de investigación que aportan al desarrollo de la misión, pero con una especial característica: trabajo de aproximación, comunicación y/o educación ambiental con la población de Bogotá.

Los proyectos y equipos que tienen trabajo directo con las comunidades de la ciudad, son convocados a un espacio de encuentro y construcción colectiva que es posible desde las perspectivas del Plan Educativo Institucional -PEI- (Rodríguez y Molano, 2007), formulado por la Subdirección Educativa y Cultural. Dicho espacio es el Seminario Permanente de Formación de Formadores Ambientales Enrique Pérez Arbeláez -SEPA-, que tiene como objetivo orientar a la reflexión sobre los fundamentos educativos que sustentan la educación ambiental ofrecida a la ciudad desde el JBB, y sobre todo a su transformación permanente según las necesidades de los contextos (LIIPGA, 2009). Durante el proceso de siete mese en el Seminario, se abordan aspectos interculturales, filosófico-epistemológicos, pedagógicos, curriculares y didácticos que pueden fundamentar la educación ambiental, con especial énfasis en la reflexión sobre las experiencias de los equipos de las tres subdirecciones con las comunidades, y un acompañamiento específico que hace alguno de los tutores u orientadores del espacio de formación de formadores ambientales.

A continuación se abordará uno de los conceptos centrales enunciados en el Plan Educativo Institucional y que es justo redimensionar, ampliar y profundizar, sobre todo porque es uno de los fundamentos que argumenta el sentido del Seminario, así como el sentido de los demás procesos de educación ambiental de la institución. Posteriormente, en el desarrollo del artículo, se hacen algunas precisiones metodológicas y se presentan las evidencias del proceso de reflexión, autoevaluación y perspectivas hacia la transformación de los procesos. Finalmente, se exponen unas reflexiones sobre las evidencias y se hacen comentarios finales que, más que finales, plantean nuevos elementos para seguir pensando la educación ambiental para una ciudad como Bogotá.

\section{LA AUTO E ÍNTER-ESTRUCTURACIÓN DE LOS PROCESOS}

Desarrollar procesos de educación ambiental dirigidos a la ciudadanía en general, obliga a pensar la educación ambiental desde otras perspectivas. En este sentido, Novo (1996) plantea que la crisis ambiental actual demanda un sistema educativo coherente y abarcador; por lo cual, la educación ambiental no puede limitarse únicamente al sistema formal, sino que también debe extenderse al sistema no formal e informal; de tal manera que se asegure la no exclusión de ningún sector o actor social, para que los ciudadanos estén en permanente reflexión sobre lo que significa la sustentabilidad.

Para lo que plantea la autora es necesario pensar en otros enfoques de la educación ambiental que se salgan de lo tradicional, exclusivamente centrado en lo académico y conceptual de las disciplinas; lo que conlleva a metodologías diferentes, que respondan a la necesidad de autoformación permanente de las comunidades, a la contextualización de los procesos educativos y su transformación, a la integración entre reflexión y acción, al reconocimiento de otras formas de conocimiento y a superar el antropocentrismo y la visión utilitarista que ve lo natural como recursos a explotar (aunque se suavice con expresiones como "sosteniblemente").

Respecto a lo anterior, es posible citar varios referentes teóricos que aportan a hacer una interpretación de la educación ambiental, sin con ello decir que se limitan las posibilidades o que se desconocen los procesos empíricos de las comunidades y sus logros frente a las transformaciones ambientales en sus territorios.

Así, el constructivismo presenta elementos epistémicos que permiten reconocer al sujeto y sus formas de modelar los fenómenos de la naturaleza. El sustento está en la declaración de la imposibilidad de conocer la realidad, y por ende de establecer una sola verdad; por lo que el conocimiento se asume como una construcción humana y social, es siempre algo convenido, propuesto, observado, inventado, acordado, relativo y surge en las comunidades de especialistas (Schweizer, 2007). Sin embargo, el constructivismo como fundamento de un proceso educativo se evidencia cuando se asume que el conocimiento que circula entre los participantes, es una construcción activa de los sujetos, que se constituye como un modelo más para representar e intervenir el mundo, y que en su proceso de construcción pasa de una subjetividad individual a una ínter-subjetividad o subjetividad consensuada (Gallego y Pérez, 1997). 
Por otro lado, pero en sintonía, los fundamentos de la complejidad permiten hablar de procesos educativos que emergen de los contextos. En este sentido, para Tovar-Gálvez y Cárdenas (2010) la educación es un fenómeno que emerge de las formas de relación que existen entre los sujetos (como componente social) y entre los sujetos y el sistema bio-físico. Con lo anterior, surge la necesidad de pensar la transformación de la educación a través del cambio de las relaciones existentes entre los componentes de los sistemas; por lo que las propuestas de educación deben nacer desde las características y condiciones de las comunidades, desde las necesidades locales y con un gran énfasis en los procesos endógenos. Ahora bien, es posible pasar de pensar la educación como fenómeno emergente a un sistema, para de esta manera atribuirle características que son deseables para los procesos de educación ambiental para la ciudadanía; los que en términos de Malinowski (2009) son: auto-organización, auto-gestión, auto-evaluación, autoinvestigación y auto-definición.

Retomando los dos referentes anteriores, es posible asumir la auto e ínter-estructuración de los procesos (para este caso de educación ambiental) como la dinámica mediante la cual los enfoques, procesos, conocimientos y acciones se generan desde el intercambio y vivencias de los actores educativos, en el marco de las condiciones de su contexto. La educación es flexible y los procesos se transforman permanentemente y de manera endógena (auto), sin negar el intercambio con otros contextos (ínter). Por ello no es posible tener certeza de lo que sucederá en los procesos.

La auto e ínter-estructuración es una alternativa coherente con el discurso desarrollado hasta ahora, en la medida que no se impone una sola visión o enfoque; más bien le apunta a la lectura de las realidades desde diversos puntos, al intercambio de conocimientos y experiencias, a la transferencia de tecnologías y a la construcción entre sujetos de manera horizontal, permitiendo modelar propuestas más acordes con las comunidades y no estandarizadas.

En tanto lo anterior, el Modelo Pedagógico para la Formación Ambiental del Jardín Botánico de Bogotá (Rodríguez y Molano, 2007) se centra en la auto-estructuración del conocimiento por parte del sujeto y las comunidades, reconociendo su pasado cognitivo y afectivo y sus experiencias, así como las posibilidades que tiene para aportar a su proceso formativo desde las peculiaridades de su contexto. Es decir, que para el Jardín Botánico los conocimientos, los procesos de educación ambiental y las acciones o experiencias de las comunidades consecuentes con procesos educativos, se construyen en los contextos de los grupos sociales; los aprendizajes se dan como procesos idiosincrásicos, de manera individual y colectiva, como construcción conjunta y permitiendo el diálogo de diversas posturas.

Entonces se asume que, según las finalidades y diversas configuraciones de los espacios de participación (para el caso del quehacer educativo del Jardín Botánico: conferencias, recorridos guiados por el Jardín, programas de formación de docentes, programas de formación de líderes ambientales, talleres lúdicos para niños, trabajo con comunidades vulnerables, sensibilización y apropiación en torno a la arborización urbana, transferencia tecnológica, entre muchos otros), es necesario reconocer las divergencias existentes, la heterogeneidad de las poblaciones y demás condiciones de los contextos educativos. Estos mismos son algunos fundamentos del Seminario, son parte de los fundamentos que se ponen a discusión en el Seminario y se espera que aporten a la transformación de los procesos de educación ambiental orientados por los profesionales del Jardín Botánico de Bogotá.

\section{METODOLOGÍA}

El enfoque metodológico es esencialmente cualitativo, por lo cual tiene varios momentos descriptivos del quehacer de los participantes y otros interpretativos desde la perspectiva del autor (a su vez orientador del proceso educativo a estudiar); así se busca acercarse a los sentidos de los actores del proceso y construir de manera inductiva formas emergentes de comprenderlos (Calvo, 1992; Quecedo, 2002; Peñaranda, 2004). En este sentido se pretende, por una parte, describir algunos elementos de la forma en que los participantes entienden los procesos de educación ambiental que lideran con las comunidades de Bogotá, y por otra, reflexionar en torno a estos, principalmente desde los fundamentos de la auto e ínter-estructuración de los procesos.

Es preciso hacer explícito que la reflexión que los participantes logran sobre los procesos de educación ambiental que lideran, es esencial para la transformación de su práctica educativa; así mismo, que la auto-evaluación es entendida como una oportunidad de interpretar un contexto o fenómeno y sobre todo de transformarlo. A través de la información cualitativa, emergente de los espacios de discusión entre los participantes y el orientador, recogida por medio de estrategias como el diario de campo y guías de registro, se evidencia la reflexión y la auto-evaluación. 
Finalmente, la información muestra la propuesta de cambio de los programas de educación ambiental que construyeron los participantes.

Cuando se hace referencia a los participantes, se debe tener en cuenta que aunque el Seminario Permanente de Formación de Formadores Ambientales Enrique Pérez Arbeláez, convocó aproximadamente a 50 profesionales de las tres subdirecciones del Jardín Botánico, aquí solo se presenta el caso de los participantes pertenecientes a la Subdirección Científica, de los proyectos de Restauración Ecológica y Conservación in situ, orientados por el autor del presente artículo (de la Subdirección Educativa y Cultural), en el marco de los módulos de interculturalidad, filosofía y epistemología, pedagogía, currículo y didáctica, desarrollados durante aproximadamente siete meses en 2010.

La primera parte de lo que se expone en los resultados, es un registro de la presentación que los participantes hacen de sus acciones con la población del Distrito Capital, con el objetivo de reflexionar si ese encuentro entre institución y comunidad se hace desde fundamentos e intenciones educativos específicos; así mismo, y con el mismo objetivo, se presenta la evaluación que los profesionales de la Subdirección Científica hacen sobre los documentos que contienen el sustento de las aproximaciones a la comunidad que se hacen desde sus proyectos. La segunda parte de los resultados reporta los elementos que configuran, en parte, la propuesta de transformación de los procesos de Restauración Ecológica y Conservación in situ, desde fundamentos en educación ambiental; siendo esta el producto de los aspectos construidos en todos los módulos del Seminario y en las tutorías con el orientador, en las cuáles se partió de la auto-evaluación y la reflexión sobre las experiencias ya vividas por los equipos en la ciudad. Finalmente se presenta la relatoría de un conversatorio entre los participantes y el orientador.

\section{RESULTADOS}

A continuación se presenta los resultados obtenidos, para lo cual es necesario anotar que la información presentada en el numeral 3.1.1 corresponde al registro en el diario de campo que el investigador redactó a través de varias sesiones de trabajo; siendo esta objeto de re-escritura o corrección en su redacción. La información presentada entre el numeral 3.1.2 y el numeral 3.2.6 se obtuvo a través de instrumentos de reflexión y evaluación que desarrollaron los participantes del proceso educativo y quienes son las muestra de la investigación; motivo por el cual el texto se transcribió fielmente y no fue sometido a corrección gramatical ni de estilo, siendo posible encontrar errores de la fuente. Finalmente, la información presentada en el numeral 3.2.7 fue obtenida en una relatoría redactada por el investigador; texto que si fue sometido a re-escritura y corrección de estilo.

\subsection{REFLEXIÓN Y AUTO-EVALUACIÓN}

\subsubsection{Descripción del quehacer con las comunidades: registro en diario de campo}

\section{Restauración Ecológica}

Vinculan a las comunidades a los procesos de restauración. Se hacen asesorías a través de actividades, con temas teóricos y prácticas. Tienen las presentaciones, fotografías y listas como registros. Poseen un documento que describe unas generalidades del proyecto y, muy someramente, hablan de los procesos de participación como sinónimo de asesorías técnicas y mencionan algunos pasos o recomendaciones. Respecto a la evaluación de los procesos, consideran que no lo hacen con intenciones educativas, pero sí hacen evaluaciones sobre aspectos ecológicos durante el tiempo en que el equipo desarrolla su trabajo. Sin embargo, consideran que es importante porque trabajan con la gente. Se trata de resolver preguntas técnicas y sobre lo biológico con la gente, aspecto que se hace cada mes o dos meses, durante tres años. No se registra la información que circula en esta evaluación, pero sí es importante porque permite hacer seguimiento a los ecosistemas.

\section{Conservación in situ}

No tienen un componente importante de educación o de trabajo con comunidades, pero recientemente hacen socialización de los resultados de investigación en los campos fitoquímico y bromatológico, entre otros. Caracterizan áreas con importancia para la conservación y son un puente para el proceso de restauración. Por ejemplo, hacen distribución geográfica de semillas y modelos de conectividad entre fragmentos de bosques que ameritan conservación. El trabajo con la comunidad se limita a explicarles en qué consiste el trabajo institucional, para que les permitan 
ingresar a sus predios para hacer el reconocimiento y acercamiento al área. Luego del ingreso al sector y del trabajo técnico, se hace una socialización con las comunidades, instituciones y subdirección. Dan información a entes territoriales, tales como el acueducto.

3.1.2. Auto-evaluación: matriz de análisis de los documentos que soportan las propuestas educativas

Restauración Ecológica

Cuadro 1. Auto-evaluación documento de restauración ecológica.

\begin{tabular}{|c|c|}
\hline $\begin{array}{l}\text { ASPECTO A EVALUAR DEL } \\
\text { DOCUMENTO }\end{array}$ & RESPUESTA \\
\hline $\begin{array}{l}\text { ¿Es clara la finalidad } \\
\text { educativa? }\end{array}$ & $\begin{array}{l}\text { La idea es clara, pero no está bien } \\
\text { definida. }\end{array}$ \\
\hline $\begin{array}{l}\text { ¿Dicha finalidad hace } \\
\text { referencia a la misión del JBB } \\
\text { y a los fundamentos } \\
\text { propuestos por el PEI? }\end{array}$ & $\begin{array}{l}\text { La finalidad sí hace referencia a la } \\
\text { misión. En cuanto a los fundamentos, no } \\
\text { han sido aplicados a los procesos } \\
\text { sociales. }\end{array}$ \\
\hline $\begin{array}{l}\text { ¿Dicha finalidad alude a la } \\
\text { formación de ciudadanos y a } \\
\text { un tipo de ciudad esperada? }\end{array}$ & No. \\
\hline $\begin{array}{l}\text { ¿Existen objetivos y a su vez } \\
\text { son consecuentes con la } \\
\text { finalidad educativa? }\end{array}$ & $\begin{array}{l}\text { Si, pero no hay una finalidad educativa } \\
\text { específica. }\end{array}$ \\
\hline $\begin{array}{l}\text { ¿Qué tipo de conocimientos } \\
\text { son evidentes en la } \\
\text { propuesta educativa para las } \\
\text { comunidades del D.C.? }\end{array}$ & Conceptual y de sensibilización. \\
\hline $\begin{array}{lcr}\text { ¿Cómo se orienta el proceso } \\
\text { educativo } & \text { con } & \text { las } \\
\text { comunidades } & \text { según } & \text { el } \\
\text { documento? } & & \\
\end{array}$ & $\begin{array}{l}\text { No hay lineamientos claros, se habla de } \\
\text { asesorías técnicas y sensibilización, pero } \\
\text { no está defirido. }\end{array}$ \\
\hline $\begin{array}{l}\text { ¿Qué posibilidades de } \\
\text { mejoramiento encuentra } \\
\text { tanto en el documento, como } \\
\text { en su práctica educativa con } \\
\text { las comunidades? }\end{array}$ & $\begin{array}{l}\text { Estructurar y desarrollar los documentos } \\
\text { y articularlos con el PEI. Integración con } \\
\text { las diferentes subdirecciones y en la } \\
\text { subdirección en cuanto a las } \\
\text { metodologias y a los procesos de gestión } \\
\text { (sic). }\end{array}$ \\
\hline
\end{tabular}

Conservación in situ

Cuadro 2. Auto-evaluación documento de conservación in situ. 


\begin{tabular}{|c|c|}
\hline $\begin{array}{l}\text { ASPECTO A EVALUAR } \\
\text { DEL DOCUMENTO }\end{array}$ & RESPUESTA \\
\hline $\begin{array}{llll}\text { ¿Es clara la } & \text { finalidad } \\
\text { educativa? } & & \\
\text { lat }\end{array}$ & $\begin{array}{l}\text { No está específica, definida y se hace } \\
\text { dependiendo de las necesidades que } \\
\text { resultan y dependiendo de los actores. }\end{array}$ \\
\hline $\begin{array}{l}\text { ¿Dicha finalidad hace } \\
\text { referencia a la misión del } \\
\text { JBB y a los fundamentos } \\
\text { propuestos por el PEl? }\end{array}$ & $\begin{array}{l}\text { Sí, cumplen con la misión del JBB y sí } \\
\text { cumple con algunas finalidades del PEI: } \\
\text { interpretación colectiva del territorio para } \\
\text { el análisis del patrimonio público } \\
\text { ambiental y el reconocimiento de la } \\
\text { diversidad biológica y cultural por medio } \\
\text { de un análisis de conflictos ambientales. }\end{array}$ \\
\hline $\begin{array}{l}\text { ¿Dicha finalidad alude a la } \\
\text { fomación de ciudadanos y a } \\
\text { un tipo de ciudad esperada? }\end{array}$ & $\begin{array}{l}\text { No directamente y no termina en la } \\
\text { realización de cambios de tipo } \\
\text { conceptual, procedimental. }\end{array}$ \\
\hline $\begin{array}{l}\text { ¿Existen objetivos y a su vez } \\
\text { son consecuentes con la } \\
\text { finalidad educativa? }\end{array}$ & $\begin{array}{l}\text { No como objetivos explícitos y no } \\
\text { involucra drectamente la finalidad } \\
\text { educativa. }\end{array}$ \\
\hline $\begin{array}{l}\text { ¿Qué tpo de conocimientos } \\
\text { son evidentes en la } \\
\text { propuesta educativa para las } \\
\text { comunidades del D.C.? }\end{array}$ & $\begin{array}{l}\text { El diálogo de saberes. Aproximaciones } \\
\text { desde diferentes escalas temporales y } \\
\text { espaciales. }\end{array}$ \\
\hline $\begin{array}{lcr}\text { ¿Cómo se orienta el proceso } \\
\text { educativo } & \text { con } & \text { las } \\
\text { comunidades } & \text { según } & \text { el } \\
\text { documento? } & & \end{array}$ & $\begin{array}{l}\text { Se orienta en una propuesta de } \\
\text { irvestigación en la que en diferentes } \\
\text { fases se interviene con las comunidades. } \\
\text { El proceso educativo en sus resultados } \\
\text { no es contemplado explícitamente, ni } \\
\text { como una finalidad. }\end{array}$ \\
\hline $\begin{array}{l}\text { ¿Qué posibilidades de } \\
\text { mejoramiento encuentra } \\
\text { tanto en el documento, como } \\
\text { en su práctica educativa con } \\
\text { las comunidades? }\end{array}$ & $\begin{array}{l}\text { Necesariamente hay que iniciar a } \\
\text { irvolucrar específicamente y visibilizar } \\
\text { una propuesta educativa. }\end{array}$ \\
\hline
\end{tabular}

\subsection{REFLEXIÓN Y PROPUESTA DE TRANSFORMACIÓN DE LOS PROCESOS DE EDUCACIÓN AMBIENTAL}

3.2.1. Finalidad educativa de los procesos: redacción de los participantes

\section{Restauración Ecológica}

En el proceso participativo del proyecto se busca que la comunidad conozca las actividades que desarrollan los diferentes componentes pertenecientes al JBB. La debilidad de dicha finalidad se encuentra en que las actitudes, en que las actividades se hacen de manera puntual y no se genera un proceso educativo que fortalezca el trabajo que en Restauración Ecológica hace el JBB. Se debe definir una propuesta educativa que incluya un proceso de acompañamiento con la comunidad, buscando integrar al mayor número de personas que se ven beneficiadas por las actividades adelantadas.

La finalidad educativa no solo se debe quedar en una participación puntual, sino que se debe generar un proceso continuo y acompañado durante el tiempo que permita la apropiación de la comunidad en las actividades de restauración.

La finalidad de un proceso educativo en el proyecto de Restauración Ecológica, es buscar en la comunidad la apropiación de los recursos ambientales recuperados por las actividades que lleva a cabo el JBB. Crear sentido de pertenencia para el ciudadano de las áreas conservadas y en proceso de recuperación. Facilitar los conceptos y demás herramientas conceptuales que permitan ofrecer una visión del significado de un ambiente, que contenga ecosistemas naturales conservados y la necesidad de recuperación de los ambientes alterados o degradados. Por último, generar proceso 
de participación de la comunidad en el proceso de restauración ecológica, para que se conviertan en un vehículo de multiplicación de las experiencias y conocimientos adquiridos, con el resto de integrantes de la comunidad. Adicionalmente y como parte de un proceso de doble vía, el JBB representado en el proyecto de Restauración Ecológica, recogerá conocimientos (experiencias y saberes) que ayuden en el fortalecimiento del componente educativo.

\section{Conservación in situ}

Las propuestas educativas desde Conservación in situ, no están claramente identificadas ni son específicas a los objetivos y actividades que se desarrollan. Se ven más como actividades de socialización o de participación en eventos esporádicos.

Para lograr una finalidad educativa, se podría articular a las investigaciones el reconocimiento de unas prioridades, conflictos y problemáticas de las comunidades que permitan aplicar los resultados, y la forma como se desarrollan las actividades para un reconocimiento de cómo buscar nuevas interpretaciones o visiones más completas de la importancia del valor en biodiversidad y en servicios de áreas bien conservadas.

\subsubsection{Tipo de ciudadano: redacción conjunta de los participantes}

La formación en términos educativos está enfocada a ciudadanos reflexivos, que encuentran en el cuidado del ambiente un aspecto clave para el mejoramiento de la calidad de vida de cada una de las personas. En el proceso educativo ambiental se busca estimular la cercanía de la comunidad a los temas ambientales, como medio para mejorar el entorno en el que viven. En el proyecto se busca la participación de una gran variedad de ciudadanos de diferente edad, formación educativa, experiencias, que tienen en común un interés por la apropiación de su región y la intención del cuidado de los recursos naturales. Se buscan ciudadanos participativos, que generen sentido de pertenencia y quieran compartir sus saberes con el resto de participantes de la comunidad.

\subsubsection{Tipo de ciudad: redacción conjunta de los participantes}

En cuanto al tipo de ciudad se busca que en el futuro encontremos espacios verdes, que provean a las comunidades que se encuentran en cada territorio de los bienes y servicios básicos que les permitan gozar de una calidad de vida óptima con el cuidado de los recursos naturales. Se busca que la ciudad sea un entramado de territorios, con una gama de comunidades que busquen el cuidado y mejoramiento del entorno en el que viven, basados en el cuidado del ambiente, al mismo tiempo que se convierten en multiplicadores de experiencias y modelo para la organización ambiental de otras ciudades.

\subsubsection{Concepción de aprendizaje: redacción conjunta de los participantes}

A través de las experiencias de trabajo con la población del Distrito Capital, se puede decir que las comunidades aprenden los conocimientos cuando estos van ligados a la solución de problemáticas propias de su territorio o de la región y que les afecta de manera directa o indirecta. Es decir, las personas se interesan en tener nuevos conocimientos en la medida en que estos le brinden herramientas de comprensión y solución a las problemáticas de la realidad que ellos perciben, para actuar sobre el medio en el que viven y de esta manera proveerse de mecanismos y desarrollar actividades que mejoren su calidad de vida.

Respecto a lo anterior, es posible establecer que los miembros de una comunidad se ven interesados en aprender nuevos conocimientos, cuando estos vienen acompañados de explicaciones y herramientas que les brinden en su territorio una comprensión más amplia del ambiente y les permita emprender actividades de solución de problemáticas. Para que el interés sea mayor se deben tener en cuenta los conocimientos que las personas tienen, la realidad que ellos perciben y el lenguaje que se utiliza con ellos, de manera que se pueda establecer un diálogo fluido en el que el intercambio de saberes sea enriquecedor y se dé un proceso educativo que dé como resultado aprendizajes significativos.

\subsubsection{Conocimientos a enseñar o poner en circulación: redacción conjunta de los} participantes

Respecto a las intenciones educativas que se tienen con las comunidades del D.C. y de acuerdo con el tipo de ciudadano pensado en el proyecto, se espera que en el aspecto conceptual las comunidades aprendan conocimiento básicos de ecología, que les permitan tener una visión más amplia de su realidad ambiental, que les permita dar explicación a ciertos procesos que se 
desarrollan en su territorio, brindando herramientas para la solución de las principales problemáticas que afectan su entorno. En lo procedimental se busca que se desarrollen tareas sencillas de cuidado de los recursos naturales que tienen en sus alrededores, y a su vez el conocimiento que adquieren lo compartan con el resto de integrantes de su comunidad (sic). En la parte actitudinal se busca que las personas de la comunidad tengan sentido de pertenencia de su territorio, de cuidado a los recursos naturales y la participación activa para la conservación del ambiente. Teniendo en cuenta lo anterior, se puede enunciar:

Cuadro 3. Tipos de conocimiento.

\begin{tabular}{|c|c|c|}
\hline Conceptual & Procedimental & Actitudinal \\
\hline $\begin{array}{lr} & \text { Ecología } \\
\text { funcionamiento } & \text { de } \\
\text { ecosistemas } & \text { y } \\
\text { causas } & \text { de } \\
\text { degradación, } \\
\text { invasión de especies } \\
\text { exóticas, procesos } \\
\text { antrópicos romo } \\
\text { minería y cultivos } \\
\text { agrícolas. } \\
\text { organizacional para } \\
\text { organimiento } \\
\text { la sostenibilidad de } \\
\text { los procesos. }\end{array}$ & $\begin{array}{l}\text { - Proceso de } \\
\text { restauración: } \\
\text { propagación, } \\
\text { plantación, control de } \\
\text { especies invasoras y } \\
\text { manejo de } \\
\text { tensionantes. } \\
\text { - Organización } \\
\text { comunitaria. } \\
\text { - Instancias y niveles } \\
\text { de participación. }\end{array}$ & $\begin{array}{l}\text { - Reflexión e } \\
\text { interpretación de las } \\
\text { situaciones } \\
\text { ambientales. } \\
\text { - Apropiación de los } \\
\text { territorios. } \\
\text { - Participación } \\
\text { ciudadana. }\end{array}$ \\
\hline
\end{tabular}

\subsubsection{Contextos y alcances: redacción conjunta de los participantes}

De acuerdo a las poblaciones y los contextos con los que se ha venido trabajando en la ciudad, es posible generalizar los procesos educativos en tres tipos de intervalos de tiempo, lo que significa pensar en diversos alcances y estrategias: a) una sesión, b) entre dos y tres sesiones, c) más de cuatro sesiones.

En consecuencia, para cada intervalo de tiempo en sesiones de trabajo directo con la población, se establecen niveles de participación ciudadana en el proceso de Restauración Ecológica:

- Nivel 1. Veeduría Ambiental Ciudadana: la población hace seguimiento al trabajo técnico de restauración realizado y a otras zonas del territorio.

- Nivel 2. Organización Comunitaria: la población establece roles y responsabilidades específicos en el proceso de seguimiento al trabajo técnico de restauración realizado y a otras zonas del territorio.

- Nivel 3. Participación en Mesas Ambientales: la población hace parte de espacios de encuentro y participación ciudadana en las que se vinculan varios sectores de la sociedad.

\subsubsection{Reflexiones en torno a la educación ambiental para la ciudad: relatoría}

Para los participantes, es evidente el desfase entre los procesos de aproximación a las comunidades y la recolección de información y la retribución o transferencia, pues desde lo institucional es clara la visión utilitarista de la flora y su manejo desde lo inmediato. Con lo anterior, se hace referencia a la tendencia a asignar valor económico a especies a las que la comunidad no daba valor monetario o tal vez solo a nivel cultural, todo en pro de su mejor aprovechamiento; aspecto que evidencia intereses de personas, instituciones y organizaciones, lo que limita las posibilidades en los procesos educativos.

Se pone en escena la idea de la apuesta política del JBB, que debe trascender las administraciones y que permita, desde lo institucional, orientar procesos más acordes para las comunidades y reales cambios culturales. En otras palabras, la posibilidad de incidencia, de cambiar significados y sentidos sobre la biodiversidad y la participación comunitaria, significa que el JBB tiene una acción política, más que desarrollo exclusivo de técnica o de cumplimiento de metas cuantitativas; la cual debe ser orientada desde filosofías institucionales que perduren más allá del ideal inmediatista de 
una administración transitoria. Es una acción política que no solo tiene sentido InstituciónComunidad, sino que también debe emerger de las experiencias mismas de las comunidades.

Retomando aspectos organizativos de la institución, es primordial comprender cómo esto posibilita o no acciones educativas y en últimas la acción política del JBB. Es una constante la desintegración de las dependencias y equipos de trabajo en los territorios, lo que se traduce en trabajo fraccionado y descontextualizado, en retribución fragmentada a las comunidades y en la dispersión de esfuerzos. La propuesta de los participantes es pensar más en los territorios que en las subdivisiones administrativas de la institución; es decir, plantear la interpretación colectiva del territorio en la institución; dicho de otra forma, plantear como objeto de estudio el contexto desde las diversas dependencias del JBB, buscando que varios equipos de las subdirecciones piensen de manera integral una condición territorial y aporten a su transformación.

Es decir, que se requiere re-prensar el JBB y lograr la real integración de sus equipos de trabajo y subdirecciones en términos de acercamiento a las comunidades e intervención en los territorios; requiere pensar la participación de las comunidades en términos políticos y de construcción de sujeto, y no de receptores de conocimientos y técnicas llevadas por el JBB; en términos de construcción de identidad frente al ambiente.

\section{DISCUSIÓN}

\subsection{RESPECTO A LA REFLEXIÓN Y AUTO-EVALUACIÓN}

\subsubsection{Sobre el quehacer con las comunidades}

\section{Restauración Ecológica}

La aproximación a las comunidades a través del proyecto, se evidencia en el proceso de participación de las comunidades en la restauración. Sin embargo, se observa que no es clara la finalidad educativa de la participación y si es un proceso que aporte al desarrollo del objetivo del proyecto o que aporte en términos educativos a la población. Se resalta que el equipo de trabajo sigue en contacto con las comunidades participantes, pero no está estructurado en términos educativos.

\section{Conservación in situ}

Se identifican dos momentos de trabajo con comunidad: a) cuando acceden a los espacios para su caracterización como áreas con importancia para la conservación, b) cuando socializan su producción con comunidades especializadas dentro y fuera del JBB. Se considera relevante pensar en el acercamiento a las comunidades para la valoración de áreas de interés. Sin embargo, se debe pensar en un proceso más estructurado, que tenga clara la finalidad no solo del trabajo técnico en el territorio, sino también en cuanto a las comunidades.

\subsubsection{Sobre la auto-evaluación de los documentos que soportan las propuestas educativas}

\section{Restauración Ecológica}

Respecto al documento, los participantes evidencian que en el proceso es clara la finalidad educativa y que esta misma es coherente con la misión del JBB, pero que en el texto no está definida de manera explícita y que no está fundamentada según el PEI y según unas expectativas frente a la ciudad. En términos metodológicos, evidencian que existen objetivos redactados, pero que no están pensados desde una finalidad; que se intercambian conocimientos relativos a lo conceptual y la sensibilización; que la metodología enunciada se limita a las asesorías técnicas y consideran necesario estructurar las propuestas en los documentos y lograr la articulación en la subdirección y con la demás dependencias del JBB.

Se identifica primordial hacer explícitas las finalidades educativas del proceso de participación de las comunidades en la restauración, y con ello las posibles formas de lograrlas. También carece de una evaluación en términos de los logros educativos y de participación de las comunidades.

\section{Conservación in situ}


La auto-evaluación desde la revisión del documento muestra que el proceso de aproximación a las comunidades en el proyecto de Conservación in situ, no tiene definido de manera explícita la finalidad educativa de ese momento de contacto con las comunidades, y aunque las actividades que se desarrollan sí apuntan en últimas a las finalidades del PEI en términos de interpretación colectiva de los territorios, no es un proceso educativo reflexionado. En lo metodológico también evidencia la carencia de objetivos que orienten lo educativo; enuncia el diálogo de saberes como tipos de conocimientos que circulan en dicho espacio; y el trabajo de intercambio con la comunidad en el marco de la investigación de la conservación. Considera que es necesario involucrar y visibilizar el proceso educativo en el proyecto.

Se hace urgente visibilizar el proceso de aproximación con las comunidades como un proceso de carácter educativo, y definir en función de ello las finalidades educativas, así como el tipo de ciudadano que participa en ello.

\subsection{RESPECTO A LA REFLEXIÓN Y PROPUESTA DE TRANSFORMACIÓN DE LOS PROESOS DE EDUCACIÓN AMBIENTAL}

\subsubsection{Sobre la finalidad educativa de los procesos}

\section{Restauración Ecológica}

El componente de Participación de las Comunidades ha tenido una finalidad en torno a incluir a la mayor parte de actores de los territorios en el trabajo de la restauración; sin embargo, dicha finalidad no es explícita en las propuestas y es necesario avanzar en la estructuración de un proceso que supere el desarrollo de actividades puntuales y que aporte al acompañamiento de las comunidades, que permita su apropiación de las actividades de restauración.

La finalidad que se propone, apunta no solo al activismo o desarrollo de actividades específicas en restauración por parte de las comunidades cuando los profesionales del JBB les convocan, sino que proyecta un proceso de reflexión, construcción de referentes pertinentes, la misma participación en las acciones puntuales de restauración ya enunciadas, el acompañamiento de la institución, y sobre todo a que las comunidades desarrollen procesos de educación comunitaria. Finalmente, se plantea un elemento que es decisivo e innovador en cuanto a la visión de los procesos educativos en el JBB, y es la sistematización de las experiencias y saberes puestos en escena durante los procesos con la comunidad, como insumo para el fortalecimiento de los procesos mismos; es decir, investigación educativa.

\section{Conservación in situ}

Retomando las reflexiones presentadas, es evidente que no es clara la intencionalidad educativa de las aproximaciones a la comunidad que el equipo realiza, por lo que la propuesta no está estructurada y se limita a una serie de actividades.

Los trabajos con la comunidad se han enfocado a las necesidades y metas del JBB como institución, por lo que considera que es necesario pensar en procesos que reconozcan los conflictos, prioridades y problemáticas de las comunidades, que signifiquen una participación activa y central de las comunidades; es de esta forma en que realmente se puede llegar a nuevas y más coherentes alternativas de interpretación sobre la importancia del valor en biodiversidad.

Así mismo, a través del trabajo reflexivo en el Seminario, es relevante y necesario pensar en la integración de las aproximaciones a la comunidad en el interior de la subdirección y con otras dependencias del JBB.

\subsubsection{Sobre el tipo de ciudadano}

La propuesta planteada tiene coherencia interna al conceptualizar: al ciudadano como sujeto reflexivo, a la apropiación de la región y la calidad de vida de las comunidades como finalidad, y a la educación ambiental como forma de lograr que las comunidades se aproximen a reflexionar sobre dicha calidad de vida. De esta manera, la propuesta se ubica en una postura centrada en el sujeto y sus posibilidades; en la apropiación de la región y en la calidad de vida, coherente con los pilares de la finalidad sugerida por el Plan Educativo Institucional; y en la educación como un proceso o medio a través del cual lograr aproximarse a los objetivos propuestos.

Cuando la propuesta se adentra a pensar la ciudad, se hace en términos ecológicos de la región y de la calidad de vida que se le puede ofrecer al ciudadano; sin embargo, se vuelve a centrarse en la 
visión activa y central del ciudadano y su responsabilidad en el cuidado y mejoramiento del entorno; así mismo, retoma la idea que ya había presentado respecto a lo esperado de las comunidades a través de los proceso de educación ambiental orientados por el proyecto de Restauración Ecológica: la multiplicación de experiencias o procesos de educación comunitaria.

\subsubsection{Sobre el tipo de ciudad}

La visión de ciudad que se presenta está en función de la calidad de vida de los habitantes, en términos de bienes y servicios. Aunque el enfoque de la propuesta está centrado en lo utilitarista de lo natural para el hombre, también tiene como un importante elemento ecologista la conectividad entre los territorios ambientales de la ciudad, además de considerar la participación ciudadana en la conservación y de pensar a Bogotá como un modelo para otras ciudades.

\subsubsection{Sobre la concepción de aprendizaje}

Es un importante avance, en tanto opción para la comprensión de los procesos de educación ambiental desde los contextos, pues se reconoce que el aprendizaje que logran las comunidades de los aspectos que los profesionales del Jardín Botánico llevan a los territorios, se da cuando los equipos de trabajo articulan o aplican los conocimientos llevados desde lo académico e institucional, con los problemas y situaciones vividos por las comunidades. Es de gran valor para orientar los procesos de educación ambiental, dar importancia a los conocimientos y experiencias que los ciudadanos tienen, para establecer un diálogo y trabajo conjunto.

\subsubsection{Sobre los conocimientos a enseñar o poner en circulación}

La perspectiva sobre qué tipo de conocimientos se presenta a las comunidades respecto a la conservación y restauración ecológica se ha ampliado, pues se ha superado lo exclusivo conceptual e informativo sobre aspectos ecológicos y se ha pensado en qué tipos de procesos, procedimientos y quehaceres pueden ser puestos a consideración para el trabajo de las comunidades, y sobre todo se hace más clara y explícita la idea de llegar a reflexiones sobre aspectos como la identidad y la participación de las comunidades en las transformaciones ambientales de sus territorios.

\subsubsection{Sobre los contextos y alcances}

La naturaleza de las intervenciones, encuentros, intercambios y procesos de educación ambiental con las comunidades del Distrito Capital es diversa. En función de ello y de la diversidad de las poblaciones, los participantes proponen una opción flexible y que busca responder a lo particular de cada caso. Con el establecimiento de los alcances de las estrategias de trabajo con las comunidades, emerge un constructo respecto a niveles de participación ciudadana.

\subsubsection{Sobre las reflexiones en torno a la educación ambiental para la ciudad}

Si bien es cierto que aún es necesario avanzar más en la estructuración de la propuesta, que es necesario madurar más ideas al consolidar algunos fundamentos y llevarlos a lo práctico de la educación ambiental, es bastante relevante lo que se lee en la relatoría de las reflexiones sobre el tema. Esto permite ver que aunque se debe seguir trabajando en la transformación de las propuestas educativas de cada proyecto, los participantes están llegando a la reflexión sobre los contextos de la ciudad, las limitaciones institucionales y a otras perspectivas para la educación ambiental. Así mismo, se puede evidenciar que la reflexión se convierte en un proceso de autoevaluación al pensar desde sus acciones específicas con la comunidad hasta la misma configuración del Jardín Botánico como institución para la ciudad.

\section{COMENTARIOS FINALES}

Con los resultados reportados, se puede evidenciar la reflexión y auto-evaluación que logran los participantes del Seminario sobre los procesos de aproximación a las comunidades de Bogotá, teniendo como eje orientador pensar qué fundamentos e intenciones educativas se tiene para ello. Esta misma información, permite ver que se va haciendo cada vez más clara la necesidad de problematizar la educación ambiental frente a los contextos, las experiencias de los participantes y las comunidades y las condiciones institucionales. 
Lo anterior, argumenta la necesidad de seguir posibilitando espacios de encuentro para intercambiar experiencias, problemas, perspectivas, fundamentos y alternativas en educación ambiental; pero sobre todo, para poner en escena las necesidades y condiciones de los territorios y las comunidades de la ciudad, los que en últimas constituyen la misión institucional

Por otro lado, la discusión o análisis sobre los resultados presentados, permiten, desde la perspectiva del autor-orientador, comprender las reflexiones y posibilidades de transformación que lograron los participantes de la Subdirección Científica, hasta el momento en el Seminario. Con ello es importante ver cómo se logran avances importantes en la propuesta, al establecer expectativas respecto a los fundamentos educativos y su puesta en escena, desde la necesidad de reconocer los contextos, las condiciones de las comunidades y las formas en que el Jardín Botánico llega a los territorios ambientales de Bogotá. Ahora bien, aunque se evidencia una propuesta amplia, pero en construcción, es de resaltar las reflexiones a las que se llega respecto a la educación ambiental para la ciudad, y que se evidencian en el discurso con mayor sentido de co-responsabilidad, participación ciudadana, sentido de pertinencia y sentido político al que llegan los participantes del Seminario.

Finalmente, es pertinente notar la relación casi que natural entre la reflexión y la auto-evaluación como promotores de transformación, y el reconocimiento de la necesidad de orientar los procesos de educación ambiental desde los contextos; es decir, que dar valor a las particularidades de las comunidades en los territorios de Bogotá, permite pensar en que las propuestas de educación ambiental se construyen y estructuran desde las experiencias de las mismas comunidades, por supuesto, en este caso orientadas por los equipos del Jardín Botánico.

\section{REFERENCIAS}

- Calvo, Beatriz. (1992, julio). Etnografía de la educación. Revista Nueva Antropología, XII. Obtenido el 26 de octubre de 2010, desde http://redalyc.uaemex.mx/redalyc/src/inicio/ArtPdfRed.jsp?iCve=15904202

- Gallego-Badillo, Rómulo, y Pérez Miranda, Royman. (1997). La enseñanza de las ciencias experimentales. El constructivismo del caos. Bogotá: Magisterio, mesa redonda.

- Jardín Botánico de Bogotá José Celestino Mutis. (n.f.). Misión Institucional. Obtenido el 6 de agosto de 2010, desde http://www.jbb.gov.co/jardinbotanico/index.php?option=com_content\&view=article\&id=39\& Itemid $=55$

- Línea de Innovación e Investigación Para la Gestión Ambiental -LIIPGA-. (2009). Seminario Permanente de Formación de Formadores Ambientales Enrique Pérez Arbeláez. [Documento de trabajo]. Jardín Botánico José Celestino Mutis, Bogotá, Colombia.

- Malinowski, Nicolás. (2009). Perspectiva histórico-comparativa, planteamiento cronológico de la reflexión paradigmática. [Documento de trabajo]. Multiversidad Mundo Real Edgar Morin, Sonora, México.

- Novo, María. (1996). La Educación Ambiental formal y no formal: dos sistemas complementarios. Revista Iberoamericana de Educación, 11. Obtenido el 20 de agosto de 2010, desde http://www.rieoei.org/oeivirt/rie11a02.pdf

- Peñaranda, Fernando. (2004, julio-diciembre). Consideraciones epistemológicas de una opción hermenéutica para la etnografía. Revista Latinoamericana de Ciencias Sociales, Niñez y Juventud, 2. Obtenido el 26 de octubre de 2010, desde http://redalyc.uaemex.mx/redalyc/src/inicio/ArtPdfRed.jsp?iCve=77320206

- Quecedo Lecanda, Rosario. (2002). Introducción a la metodología de la investigación cualitativa. Revista de Psicodidáctica, 14. Obtenido el 22 de octubre de 2010, desde http://redalyc.uaemex.mx/redalyc/src/inicio/ArtPdfRed.jsp?iCve=17501402

- Rodríguez, Tania, y Molano, Carolina. (2007). Plan Educativo Institucional del Jardín Botánico José Celestino Mutis. Bogotá: Imprenta Nacional.

- Schweizer, Stefan. (2007). En historia de las ideas, la pedagogía constructivista proviene del idealismo alemán. Electroneurobiología, 15(4). Obtenido el 6 de agosto de 2010, desde http://electroneubio.secyt.gov.ar/Stefan_Schweizer_Pedagogia_ constructivista_idealismo_aleman.pdf

- Tovar-Gálvez, Julio César, y Cárdenas Puyo, Nhora. (2010). Formación inicial de docentes en el marco de las actuales perspectivas en investigación educativa: la complejidad. En Memorias IV Congreso de Transdisciplinariedad, Complejidad y Ecoformación. Universidad de Costa Rica. Obtenido el 10 de junio de 2010, desde: http://congreso.samisolutions.com/attachments/066_Tovar_Cárdenas_Formación\%20Doce nte\%20(Transfisciplina\%20y\%20Auto-Eco-Orgnización3).pdf 
1. Coordinador del Programa de Formación de Líderes Ambientales y tutor del Seminario Permanente de Formación de Formadores Ambientales Enrique Pérez Arbeláez, Subdirección Educativa y Cultural del Jardín Botánico de Bogotá José Celestino Mutis. 